\title{
Procès-verbal de la séance extraordinaire de la Chambre médicale ${ }^{1}$
}

\author{
Samedi 26 juin 2004, de 9h30 à 17h00, Palais des congrès à Bienne
}

\section{Annamaria Müller Imboden}

1 Remarque de la rédactrice du procès-verbal: les élections prévues au point 3 sont conduites parallèlement au traitement des points 4 à 10 . Pour des raisons de lisibilité, le procès-verbal suit l'ordre du jour.

2 Remarque de la rédactrice du procès-verbal: le texte des discours se trouve dans un chapitre séparé, en fin de procès-verbal.

3 Cette période administrativ va de la Chambre médicale du 26 juin 2004 à la Chambre médicale ordinaire de 2008.

4 Art. 47, $1^{\text {er }}$ al.: «Le CC se compose du président, de deux vice-présidents et de 6 à 8 membres.»

5 Art. 47, 2e al.: «Lors de l'élection des membres du CC, il doit être tenu compte de façon appropriée des différentes langues nationales, des région et des divers domaines de la profession médicale. Le CC comprend dès lors au moins deux représentants de la Suisse romande (au moins trois pour onze membres) et un représentant du Tessin. L'un au moins des membres du CC est aussi membre de l'ASMAC et un autre, membre de l'AMPHS.»

Début de la séance: 9h35.

\section{Accueil, communications, constitution du Bureau}

Hans Heinrich Brunner, président de la FMH, souhaite la bienvenue aux participants à la séance extraordinaire de la Chambre médicale à Bienne et constate que l'assemblée atteint le quorum nécessaire. Il donne quelques indications d'ordre technique et d'autres concernant les dispositions de sécurité. Il salue les hôtes présents, à savoir Jean-Frédéric de Montmollin pour le travail de relations publiques (point 5) et François Bossard pour la commission d'évaluation AA/AM/AI (point 6).

Le président communique les noms des scrutateurs. Il s'agit de Jean-Pierre Grob, Urban Laffer, Hanswilly Mattes, Rolf Naegeli, Martin Rüegger, Dominique Schmidt, Ulrich Seefeld, Hans Anton Vogel, Franziska Zogg.

\section{Communications du président}

Le président sortant jette un bref coup d'œil rétrospectif sur sa présidence et remercie le corps médical pour sa confiance et son soutien, mais aussi pour la critique constructive qui lui a été communiquée durant toute cette période. Il souligne qu'il restera lié pour toujours à la FMH aussi après son départ. Des allocutions d'adieux sont prononcées par Christoph Ramstein, président du VEDAG, Ursula Steiner-König, vice-présidente de la FMH et Yves Guisan, qui s'exprime en particulier aussi au nom du corps médical de la Suisse romande. L'activité de H. H. Brunner est honorée par le Dr Marc Baur, Bâle, délégué le plus «anciennement élu» à la Chambre médicale. ${ }^{2}$

\section{Elections générales pour la période administrative 2004-20073}

La proposition $n^{\circ} 3 / 1$ (Comité central) est conforme aux statuts ${ }^{4}$ et concerne le nombre de membres à élire au Comité central (CC) pour la prochaine période administrative, c'est-à-dire 9, 10 ou
11 membres. Compte tenu du rapport des voix, le nombre 10 cause problème. Un comité à 11 membres garantit aux régions linguistiques, disciplines médicales et sexes d'être représentés de manière équitable. Une diminution permanente du Comité central serait éventuellement à prévoir dans le cadre de la réforme des structures. Les délégués acceptent la proposition visant à élire 11 membres et non pas 9 au Comité central. Le nombre de membres au Comité central est fixé à 11 membres par 163 voix contre 11, sans abstention.

\subsection{Election du Comité central de la FMH}

Se représentent: Max Giger, Yves Guisan, LudwigTheodor Heuss, Franco Muggli, Marie-Christine Peter-Gattlen, Ursula Steiner-König, Susanna Stöhr. Se présentent pour la première fois: Jacques de Haller, Pierre-François Cuénoud, Olivier Kappeler, Erich E. Küng, Richard Nyffeler, René Raggenbass. Tous les membres déjà en fonction ainsi que Jacques de Haller, Pierre-François Cuénoud et Olivier Kappeler sont élus au premier tour. René Raggenbass est élu au quatrième tour. Le Comité central est ainsi au complet et les exigences relatives à une représentation équitable des intérêts sont remplies. ${ }^{5}$

\subsection{Election du président de la FMH}

Sont candidats: Max Giger, Yves Guisan, Jacques de Haller et Ludwig-Theodor Heuss. Une motion d'ordre de Guy Jenny, ophtalmologie, concernant la prise de connaissance des conditions d'engagement des candidats à la présidence, est rejetée sans comptage des voix. Au cinquième tour, la Chambre médicale élit par 101 voix Jacques de Haller président de la FMH, qui succède ainsi à Hans Heinrich Brunner. Jacques de Haller accepte son élection avec joie et souligne que, durant son mandat, il veillera tout particulièrement à créer un capital confiance, à intégrer tous les groupements et à réaliser la réforme des structures. Le président sortant remet à son successeur nouvellement élu une «clé symbolique de la $\mathrm{FMH}$. 


\subsection{Election de la vice-présidente et du vice-président de la FMH}

Après une brève interruption de la séance, le Comité central nouvellement élu propose de réélire les deux vice-présidents en fonction jusqu'à présent, à savoir Yves Guisan et Ursula Steiner-König. Les deux candidats sont confirmés au premier tour.

\subsection{Election de remplacement éventuelle du président de la CFPC FMH}

Il est renoncé à une élection de remplacement à la tête de la CFPC car Max Giger, qui en a été élu président à la Chambre médicale ordinaire, peut conserver son mandat.

\section{Réforme des structures de la FMH}

Ludwig-Theodor Heuss présente le dossier qu'il a repris le 29 avril 2004, conjointement avec Franco Muggli, du président sortant. Ce thème est de première importance pour l'avenir de la FMH. Il n'a pas été traité à la Chambre médicale ordinaire du 29 avril 2004 par manque de temps. La structure de la FMH a déjà été revue dans les années nonante. Pour être en mesure de relever les défis du futur, il est toutefois nécessaire de procéder à une réforme approfondie, qui doit viser à un but précis. Par conséquent, il faut tout d'abord définir l'orientation stratégique de la $\mathrm{FMH}$, ce qui ne pourra se faire qu'avec la participation de tous les groupements concernés, sans oublier la base. Pour y parvenir, la proposition $n^{\circ} 4 / 1$ (Ramstein, Soleure) suggère une «Conférence réunissant tous les groupes» sous la direction d'une organisation de conseil externe. Cette proposition est soutenue par le Comité central. Les propositions $n^{\circ} 4 / 2$ (Favrod-Coune, Vaud) et $n^{\circ} 4 / 3$ (Schlup, Berne) vont également dans le sens d'une réforme des structures mais en suggérant une procédure différente. La proposition Schlup reprend donc la proposition Favrod-Coune en la modifiant.

La discussion qui s'ensuit porte sur les points suivants:

- une mise au concours devrait avoir lieu pour l'accompagnement externe;

- les organisations de base doivent être représentées de manière appropriée;

- une prise en compte adéquate doit être accordée à la base, c'est-à-dire aux membres;

- la composition des groupes doit être clairement établie et communiquée;

- le calendrier doit être fixé de manière réaliste;

- les rôles et tâches des organes actuels (Chambre médicale, Conférence des prési- dents, Comité central) dans le processus des réformes, présentés différemment dans les propositions, doivent être harmonisés;

- des ressources suffisantes doivent être mises à disposition en temps, personnel et finances pour le projet.

Les propositions ( $n^{\circ} 4 / 1$ à 4/3) sont retirées en faveur de la proposition 4/4 (Favrod-Coune, Ramstein, Schlup, Heuss). Celle-ci demande de lancer un processus visant à mettre en place une réforme des structures qui recueille l'adhésion de tous les groupements et réponde aux besoins d'une association moderne. Ce processus se fondera sur une analyse différenciée des objectifs, besoins et options stratégiques de la FMH et sur une large participation de la base. Les initiateurs, avec Franco Muggli, forment un groupe de pilotage qui présentera à la Conférence des présidents une proposition concernant la suite de la démarche. La Chambre médicale prendra la décision définitive. Cette proposition est acceptée sans comptage des voix.

\section{Concept de relations publiques de la FMH}

Max Giger, qui a également repris le 29 avril 2004 le dossier du président sortant à titre intérimaire, constate que le concept n'a subi aucun changement. Ici aussi, il s'agit maintenant de formuler tout d'abord des visions, des stratégies et des objectifs avant de délibérer sur les structures et l'organisation des relations publiques. Ce processus suivra donc, par la force des choses, celui de la réforme des structures. Jean-Frédéric de Montmollin informe sur l'origine, le contenu et l'effet du Manifeste de la SMSR «Neuf pistes». Christoph Ramstein signale que le VEDAG a également mis au point des principes directeurs selon une procédure semblable à celle proposée pour la réforme des structures. Ces principes vont être fixés dans un document qui sera publié sous peu.

La proposition $n^{\circ} 5 / 1$ (Comité central) proposant d'approuver le concept de relations publiques de la FMH (version 3.0) est acceptée par 156 voix sans opposition et avec 12 abstentions.

La proposition $n^{\circ}$ 5/2 (Jenny, ophtalmologie) demande au Comité central d'élaborer un concept de politique de la santé selon une vision propre au corps médical. Comme il s'agit d'une proposition concernant les travaux préparatoires stratégiques sur la réforme des structures, elle est transmise au Comité central sans vote préalable. 


\section{TARMED}

Res Haefeli, président du G7, informe sur les affaires actuelles du bureau du TARMED. Il s'occupe essentiellement des travaux préparatoires relatifs aux thèmes qui devront être traités ces deux prochaines années par la FMH, parmi lesquels il y a aussi les propositions du G7 pour l'organisation du domaine tarifaire. Il fait aussi mention du rapport que le Préposé fédéral à la protection des données a publié au sujet du TARMED. Malgré l'aspect positif de ce rapport pour le corps médical, Haefeli regrette les considérations d'ordre général sur le code de diagnostic «tessinois» utilisé dans le domaine ambulatoire.

Urs Stoffel, membre du Conseil d'administration de NewIndex et du bureau de la neutralité des coûts, constate avec plaisir que les «médecins indépendants ont accompli leur devoir». Il l'explique en donnant des précisions sur le mode de fonctionnement du Bureau de la neutralité des coûts et les premiers résultats obtenus. Les données fournies par la FMH sont représentatives et reconnues par les partenaires tarifaires.

François Bossard fait le point sur les travaux de la commission d'évaluation Tarif AA/AM/AI dans le contexte de la stabilité des coûts par cas. Pour le moment, la valeur du point tarifaire reste inchangée et aucune nouvelle mesure ne sera prise. En revanche, on envisage de prolonger la phase prévue pour la stabilité des coûts par cas. Concernant les chiffres de la FMH, il existe encore des problèmes de coordination que le Comité central fraîchement élu devra résoudre.

Il ressort de la discussion qui suit que la protection des données, le secret médical et la transparence exigée par les assureurs, en particulier concernant le diagnostic, génèrent un énorme champ de tensions. Dans son rapport, le Préposé à la protection des données semble avoir été trop peu clair et les personnes qui doivent appliquer ses directives éprouvent une insécurité désagréable. La nécessité d'agir revient maintenant à la politique, c'est-à-dire au Parlement et au Conseil fédéral. retirée.

La proposition $n^{\circ} 6 / 1$ (Jenny, ophtalmologie) est

\section{Code de déontologie - Approbation de directives de I'ASSM}

a) Directives médico-éthiques concernant les examens génétiques sur l’homme (1998); b) Directives médico-éthiques pour la transplantation de tissus fotaux humains (1999);

c) Directives concernant les problèmes éthiques aux soins intensifs (1999);

d) Principes médico-éthiques concernant les xénotransplantations (2000);

e) Directives médico-éthiques pour l'exercice de la médecine auprès de personnes détenues (2002).

La proposition $n^{\circ} 7 / 1$ (Comité central), qui demande d'insérer les directives susmentionnées de l'ASSM dans le Code de déontologie, est acceptée sans comptage des voix ni opposition et avec 2 abstentions.

\section{Formation postgraduée et continue}

\subsection{Création de titres, formations approfondies et attestations de formation complémentaire}

\section{a) Création d'une attestation de formation} complémentaire en psychothérapie déléguée

En l'absence de quorum, le point 8.1 a été traité à la Chambre médicale du 29 avril 2004 uniquement sous forme consultative. La proposition $n^{\circ}$ 8.1/1 (Comité central) portant sur la création d'une attestation de formation complémentaire en psychothérapie déléguée est acceptée sans comptage des voix, avec 5 oppositions et 9 abstentions.

\subsection{Révision de la Réglementation pour la formation postgraduée}

Max Giger, président de la CFPC, communique que les délégués à la Chambre médicale ont approuvé la révision de la RFP par le fait qu'aucune opposition n'a été exprimée à l'encontre de ladite révision.

\subsection{Révision de la Réglementation pour la formation continue}

La proposition $n^{\circ}$ 8.3/1 (Muff, Zurich) souhaite la reconnaissance, à hauteur de 10 crédits au maximum, de sessions non spécifiques portant sur l'éthique professionnelle, la politique de la santé, les questions de gestion ou la formation dans le domaine des urgences. Cette proposition est acceptée sans comptage des voix, par 3 oppositions et 5 abstentions. 


\subsection{Société suisse de médecine du sport: droit de siéger à la CFPC}

La proposition $n^{\circ} 8.4 / 1$ (Villiger, médecine du sport) demande que la Société suisse de médecine du sport (SSMS) obtienne le droit de siéger à la Commission pour la formation postgraduée et continue (CFPC). Elle est acceptée par 81 voix contre 26 et 17 abstentions.

\section{Révision des statuts (organisations des médecins d'hôpitaux AMPHS/ASMAC)}

Hans-Ueli Würsten, AMPHS, et Peter Studer, ASMAC, présidents des organisations de médecins concernées font le point de la situation. Différents entretiens ont eu lieu entre les organisations, y compris les sections sécessionnistes de l'ASMAC Genève (AMIG) et de Neuchâtel (AMINE). Ces entretiens visaient à mettre en évidence les points communs. Les efforts de rapprochement ne doivent toutefois pas être sous-estimés car il s'agit d'un «cheminement progressif». Pour cette raison, ce thème doit être régulièrement mis à l'ordre du jour des séances afin que l'on puisse informer les délégués sur l'évolution de la situation.

\section{Divers}

Pour des raisons juridiques, il est impossible de traiter la proposition $n^{\circ}$ 10/1 (Röthlisberger, Grisons) portant sur les accords bilatéraux, car la décision en la matière ne relève pas de la compétence de la Chambre médicale. Elle est donc supprimée en conséquence.

Hanspeter Kuhn, responsable du service juridique, communique un changement de personnes au secrétariat. Une information ad hoc paraîtra dans le Bulletin des médecins suisses.

Rudolf Wissmann, St-Gall, informe sur la suppression du crédit octroyé à l'organe de coordination des médecins de premier recours par le Grand Conseil saint-gallois et le regrette vivement.

La séance prend fin à 16h45.

\section{Allocutions}

Allocution de départ du président de la FMH sortant, le Dr Hans Heinrich Brunner

Chers collègues

L'occasion m'est donnée de prendre congé de vous. Vous m'avez élu président de la FMH, il y a dix ans ou presque, et le temps des adieux est arrivé. Je ne vais pas me lancer dans une longue et fastidieuse description de ces années passées. Mon propos se veut court, sous la forme d'un compte-rendu, avant de nous quitter dans le respect des uns et des autres.

Cette dernière décennie, j'ai poursuivi quatre objectifs avec une constance quasi permanente. Le premier était d'une importance certaine - et l'est demeuré quels qu'en aient été les aléas maintenir l'unité de la FMH. Je laisse à d'autres le soin de juger de ma présidence. La Chambre médicale de ce jour me montre néanmoins que certains objectifs ont été atteints. J'ai tenté également de développer une association de type très corporatif en une organisation professionnelle moderne, de manière à lui donner un meilleur ancrage, sur le plan politique et sociétal, en Suisse comme à l'étranger. C'est un processus de longue haleine que je souhaite à mes successeurs de poursuivre. J'ai aussi tenté d'ouvrir de nouveaux débouchés stratégiques. Je ne les nommerai pas tous, sinon peut-être les plus importants. Ce qui m'a toujours importé au plus haut point, vous le savez, c'est le recours aux potentiels en présence et leur réalisation à la lumière des technologies de l'information. Je veux surtout parler d'action médiatique au sens large du terme, de travail de presse en particulier, qui, je me permets de le relever, a vu certains développements ces dix dernières années. C'est un projet qui mérite d'être suivi en permanence, en concomitance avec le savoir en matière d'économie et de tarifs. Une dernière résolution a été de maintenir une FMH indépendante et en bonne santé financière. Certains succès ont été obtenus sur cette voie.

On ne saurait poursuivre ces objectifs sans s'ouvrir au débat politique et sociétal et sans craindre le discours public - et l'autocritique. Vous savez que je n'ai pas craint d'exercer celleci et vous ne m'avez pas ménagé vos remarques, parfois acerbes. Je me souviens des houleux débats sur les brebis galeuses ou fameux «moutons noirs». Il est possible que je n'ai pas été un parfait lobbyiste, je veux bien l'admettre. Je ne saurais non plus exposer en détail pourquoi je fus souvent contraint (sans l'avoir cherché), de prendre seul des décisions et de les défendre contre vents et marées. Je revendique néanmoins l'avoir toujours fait au service de la $\mathrm{FMH}$, en négligeant les intérêts personnels et certaines circonstances du moment. Ces contingences, ainsi qu'une situation politique fluctuante, ont fait que les critiques à mon égard ne m'ont pas épargné. Vous ne l'ignorez pas, les levées de bouclier ont été particulièrement impétueuses 
l'année dernière. Je vous l'affirme, je ne quitte pas la FMH en adversaire vindicatif de la FMH ou de ses membres, comme on a pu le lire dans le courrier des lecteurs du Bulletin des médecins suisses. Je tiens à poursuivre les contacts, à les cultiver et à les rechercher, de manière à trouver, dans la mesure du possible, des solutions objectives et scientifiquement fondées, donc de jeter un pont entre mes anciennes et mes nouvelles activités. Une sentence de Confucius, Sage parmi les Sages: «Oublie les injures, n'oublie jamais les bienfaits» me paraît servir cette volonté tout en illustrant mon allocution de départ.

Je souhaite à la FMH, à vous tous, la sagesse et la force de résister aux tempêtes. L'époque qui s'annonce ne promet sans doute pas d'être plus facile qu'avant. Sachez que dans la mesure où mes fonctions me le permettront, vous trouverez toujours en moi un soutien fidèle.

Un dernier mot en tant que président avant de passer aux votes: un président de la FMH est faillible, comme tout un chacun. Ce qu'il entreprend doit l'être en négligeant tout intérêt personnel et financier. Sinon, il n'est pas fait pour ce métier. C'est ainsi que les personnes que vous élirez ont droit à votre respect, à l'avenir également: l'irrespect à leur égard, comme à celui de la plus haute autorité corporative n'est qu'irrespect envers l'organisation et, finalement, envers vous-même!

\section{Allocution du Dr Christoph Ramstein, président de la Société médicale du canton de Soleure}

Hans Heiri, au nom du VEDAG et de ses présidents, je tiens à revenir sur nos rencontres de cette dernière décennie. Pour ce faire, je me fonde sur certains courriels que j'ai conservés. Un premier message de toi, datant du 17 janvier 2002 est ainsi libellé: «On m'accorde de toute façon une préférence incongrue pour Soleure». Dans ces circonstances, tu ne pouvais pas te dérober, puisque né à Olten, baptisé en ces lieux et émigré plus tard en Suisse centrale, tout en restant sur le territoire du VEDAG. C'est dans cette région principalement que tu as développé ton savoir professionnel et politique, faisant partie dix ans durant du Club d'Olten avant d'être nommé président de la FMH. L'ère de ton prédécesseur s'est transformée dans un style très personnel, marqué par ton énorme force de travail, ton efficacité, ta productivité et tes formules percutantes qui ont souvent bouleversé les convenances et poussé l'interlocuteur dans ses derniers retranchements. On y a souvent $\mathrm{vu}$ ta grande érudition, tes connaissances en histoire, en art et en philosophie, ainsi que ton activité littéraire exercée au cours de tes études de médecine par l'intermédiaire de romans à sujet médical tels que «Dr. Landmann in der Entscheidung». Je pense aussi à ta manière analytique de penser, à ta persévérance, ton opiniâtreté et ton endurance, aussi bien dans le débat que dans la poursuite d'objectifs. Qui, parmi les polémistes, aurait supporté aussi longtemps de défendre un tarif tel que le GRAT, puis le TARMED, en dépit de tous les tirs de barrage essuyés au cours de cette période? Comment oublier, non plus, la maestria avec laquelle tu géras tes interventions à l'émission télévisée «Arena», quelle que soit la situation, la plus délicate soit-elle. Dans ces débats, c'est surtout la génération de l'ASMAC que tu aimas défendre. Tu as représenté notre fédération à l'extérieur, ce qui a tout son prix, vu les difficultés qu'il y a à défendre les intérêts et les opinions de tous les membres. Pour ce faire, une bonne cuirasse s'impose!

Ton talent n'est plus à faire en matière de tenue de séance, conférence des présidents ou Chambre médicale, même si certaines consœurs et confrères ont pu te reprocher une direction des débats quelque peu militaire. Tu as accordé une confiance des plus grandes à tes collaborateurs et collaboratrices, à l'Elfenstrasse comme au sein de cercles plus restreints. Je souhaite ainsi transmettre mes remerciements au secrétariat général et au secrétariat de direction pour l'énorme somme de travail accomplie pendant toute l'année.

Cher Hans Heinrich, tu as toujours été attentif à ceux qui t'ont manifesté leur estime en tant qu'homme politique de terrain, médecin et être humain. Ils ont ainsi mieux réussi à donner leur avis et tu n'as pas manqué d'intégrer et d'appliquer les idées qui pouvaient en découler. C'est avec ces mots que nous te remercions pour l'énorme travail accompli et te laissons à tes activités à l'OFSP. Qu'une foule de d'objets pratiques t'accompagne dans tes nouvelles aventures, pas tout à fait sans danger. Une pioche, p. ex. éprouvée par la LAMal, pourra te servir à révéler les problèmes en suspens, notamment les articles 32 et 56, ceux précisément que la FMH a soigneusement mis de côté et qui te reviendront désormais par la grande porte. J'y joins une copie de ma proposition de l'année 1999 dans laquelle je demandais à la FMH d'enfin aborder les critères EAE (efficacité, adéquation, économicité) en toute conscience des difficultés représentées. A l'OFSP, tu auras sans doute à traiter ces problèmes en suspens. Le prochain objet utile est une dynamo, qui devrait, grâce à sa lumière, mieux percevoir et calmer tes propres angoisses. 
Il te faudra aussi des gants ignifugés pour éviter de te brûler les doigts. Deux bonnets ne seront pas de trop non plus dont l'un devrait servir de camouflage si tu veux passer inaperçu dans la Commission de la sécurité sociale et la santé (CSSS) du Conseil national sans réveiller ceux qui dorment. Un gilet pare-balles pour éviter les éventuelles agressions des angoissés qui ne parlent que du dangereux tandem CouchepinBrunner. Un gilet de sauvetage en cas de noyade politique. Puis un réservoir à eau et sa louche, pour une sorte de buffet froid montrant symboliquement à tes ennemis qu'eux non plus n'ont pas de remède miracle. Le trépied aura aussi sa fonction, celle de t'accompagner dans tes loisirs et tes gardes de nuit à l'Hôpital de l'Ile, pour montrer comment se satisfaire de ses propres ressources et économiser ainsi des coûts. Enfin, une cravate à motifs militaires si tu te rends parfois à Weggis, où elle sera l'ornement idéal selon l'adage: «Please don't be anytime so busy, take it from time to time a little easy!»

Je tiens aussi, au nom des présidentes et présidents du VEDAG, à te remettre ce bon, pour une excursion en leur compagnie dans une ambiance qui te rappellera le club d'Olten. Nous la terminerons par un repas sympathique pris en commun. Le bon est valable d'ici les prochaines élections au CC, au printemps 2008 (applaudissements).

\section{Allocution du Dr Ursula Steiner, vice-présidente de la FMH}

Cher Hans Heinrich, je sais que tu aurais préféré te passer de compliments formulés à ton départ. Mais ce n'est guère possible. Rétrospectivement, les dix dernières années avec toi au Comité central me rappellent la situation à son tout début. Nous étions en conclave pour discuter du clivage entre l'«éthique» de la profession et FMH Services, qui devait être «rentable». Une plaisanterie se fit jour: le vocable FMH pourrait signifier «for more happiness». Dans la foulée, je pensai à tout ce que ces trois lettres pourraient encore vouloir dire, en relation avec toi, le Comité central et la FMH dans son entité. Les exemples qui suivent n'ont de sens qu'en allemand et sont une suite de jeux de mots sur ce sigle: élargir l'horizon, multiplier les hypothèses, oublier l'auréole du médecin, relever les défis, éviter les coups de Jarnac, se passer des compliments, prôner la ténacité, l'action, la recherche en médicaments, la hiérarchie, le HIN, et surtout l'humour: je me souviens du récit de ta prestation dans un studio de télévision avec le futur conseiller fédéral Blocher.

$\mathrm{Au}$ Comité central, nous souhaitions, avec toi, prôner plus d'espoir, de meilleurs honoraires, un plus grand don de soi et plus d'honnêteté, mieux faire face aux mauvaises nouvelles, assurer les hypothèques. Pour l'ensemble de la FMH, nous souhaitions une attitude plus respectueuse, plus de cordialité et finalement, plus d'inspiration. C'est cette inspiration dont tu nous a gratifiés, cher Hans Heinrich. Nous ne pouvons qu'espérer que l'avenir de la FMH avec toi comme vice-directeur de l'OFSP ne pourra que profiter de cette nouvelle situation. Nous te souhaitons dans tes nouvelles fonctions chance et prospérité. Ce ne sont certes pas les nouveaux défis qui te manqueront.

\section{Allocution du Dr Yves Guisan, vice-président de la FMH}

Mon cher Hans Heinrich, chers collègues, chers amis,

Je ne viens pas ici avec un sac postal mais avec quelque chose sans doute de plus modeste, mais assez symbolique. La Société vaudoise de médecine, les Romands et d'autres encore, se sont montrés passablement critiques et ne t'ont pas toujours rendu la vie facile. Mais cela ne les empêche pas d'avoir pour toi une estime certaine, de t'être reconnaissants pour tes compétences, ton immense capacité de travail, et surtout de tes visions que nous n'avons pas toujours très bien comprises. Mais le temps nous rattrape et nous montre bien que tu avais senti avant nous un certain nombre de choses que les événements maintenant nous démontrent.

Tu es un homme de la Suisse centrale. Une fois, le Comité central s'était réuni sur les hauteurs des alpages vaudois. Et c'est en souvenir de cette sortie à laquelle tu avais participé et éprouvé beaucoup de joie par une magnifique journée d'automne que je te remets ici un symbole de cette journée qui évoque la montagne, la sérénité mais aussi la persévérance. Je te prie de l'ouvrir, parce que je crois cela montrera assez bien à tous nos collègues ce que nous voulons dire par là, et ce que nous autres des montagnes vaudoises ont en commun avec toi, homme des montagnes de la Suisse centrale. Merci, Hans Heinrich! 